\title{
Survivin expression in hepatocellular carcinoma: correlation with proliferation, prognostic parameters, and outcome
}

\author{
Angela C Fields ${ }^{1}$, George Cotsonis ${ }^{2}$, Debbie Sexton ${ }^{1}$, Robert Santoianni ${ }^{1}$ and \\ Cynthia Cohen ${ }^{1}$ \\ ${ }^{1}$ Department of Pathology and Laboratory Medicine and ${ }^{2}$ Department of Public Health, Emory University \\ School of Medicine, Atlanta, GA, USA
}

\begin{abstract}
Survivin is a novel inhibitor of apoptosis. It is detected in fetal and neoplastic adult tissue, but not in normal tissues. Several recent studies have shown that survivin not only inhibits apoptosis, but also accelerates cancer cell proliferative activity. Expression of the protein may be of prognostic significance and therapeutic relevance in many cancers. We investigated survivin expression in hepatocellular carcinoma, correlating results with proliferation (MIB-1), prognostic factors, and outcome. Paraffin-embedded sections of 72 hepatocellular carcinoma were immunostained for survivin and MIB-1 using tissue microarray technology. Expression was evaluated in nuclei and cytoplasm as intensity (0-3+), and percentage of positive cells scored on a four-tiered system with less than $10 \%=$ negative; $10-25 \%=1 ; 26-50 \%=2 ; 51-75 \%=3$; and $76-100 \%=4$. Frequency of nuclear survivin expression was $43 \%$. There was a significant correlation between nuclear survivin expression and nuclear grade $(P=0.0271)$, microvascular invasion $(P=0.0064)$, mitotic rate $(P=0.0017)$, and MIB-1 $(P=0.0001)$, as well as local recurrence $(P=0.0487)$, and disease-free survival $(P=0.0098)$. Histologic grade $(P=0.0544)$ and stage $(P=0.0548)$ tended to correlate with survivin expression, which did not correlate with cirrhosis, tumor necrosis, multiple tumors, metastatic disease, or overall survival. Survivin expression correlates with poor prognostic parameters (high nuclear and histologic grade, microvascular invasion, increased proliferation (mitotic count, MIB-1)), local recurrence, and shorter diseasefree survival, but does not correlate with overall survival. An important role is suggested for survivin in progression, recurrence, and treatment of hepatocellular carcinoma.
\end{abstract}

Modern Pathology (2004) 17, 1378-1385, advance online publication, 11 June 2004; doi:10.1038/modpathol.3800203

Keywords: hepatocellular carcinoma; survivin; proliferation; apoptosis; prognosis

Survivin, a member of the inhibitor-of-apoptosis proteins (IAP) family ${ }^{1,2}$ is not present in most normal adult differentiated tissues. Its mRNA and the protein are present in large amounts in fetal tissue and most cancers. ${ }^{3,4}$

Survivin protein suppresses apoptosis (programmed cell death) and stimulates cell division. ${ }^{5}$ The mechanism whereby it blocks apoptosis is assumed to be via an effect on caspase-9 (causing dissociation of the Apaf-1-caspase-9 complex), which is activated through extrinsic and intrinsic pathways. $^{2,6}$ In the intrinsic cell death pathway, upstream stimuli (such as activation of p53) induce

Correspondence: Dr C Cohen, MD, Anatomic Pathology, Emory University Hospital, G144, 1364 Clifton Road, NE, Atlanta, GA 30322, USA.

E-mail: ccohe01@emory.edu

Received 29 March 2004; revised 30 April 2004; accepted 1 May 2004; published online 11 June 2004 expression of proapoptotic bcl-2 family proteins such as bax. The membrane-permeabilizing effects of bax and other proapoptotic proteins are inhibited by antiapoptotic proteins, such as bcl-2 and bcl- $\mathrm{X}_{\mathrm{L}}$, which also may inactivate caspase- 9 by binding and inactivating the adapter protein Apaf-1. ${ }^{7,8}$ When, as a result, caspase- 3 is not activated, apoptosis is inhibited. ${ }^{1,9}$

Survivin is expressed during the $\mathrm{G}_{2} / \mathrm{M}$ phase of the cell cycle. It associates with microtubules of the mitotic spindle, at the beginning of mitosis. Disruption of survivin-microtubule interaction results in loss of survivin antiapoptotic function, and during mitosis, caspase-3 activity increases. ${ }^{10,11}$ Thus, survivin seems to inhibit apoptosis of tumor cells, and accelerate their mitotic activity. ${ }^{12}$ In support of this stimulatory effect on proliferation, survivin expression is shown in hepatocellular carcinoma cell lines, to result in a decrease in the G0G1 phase with an increase in the $S$ phase. ${ }^{13}$ This promotion of cell 
proliferation by survivin was due to its interaction with cyclin-dependent kinase $4(\mathrm{Cdk} 4)$ with release of p21 (WAFI/Cip 1) from Cdk4. ${ }^{13}$ In human hepatocellular carcinoma, nuclear survivin by immunofluorescence and immunohistochemistry strongly correlated with proliferation index (Proliferation Nuclear Cell Antigen) although not with apoptotic index (Tunel method). ${ }^{13,14}$ In other studies, ${ }^{12,15,16}$ survivin messenger RNA (mRNA) quantitated by real-time reverse transcriptase-polymerase chain reaction (RT-PCR) (divided by amount of glyceraldehyde-3-phosphate dehydrogenase [GAPDH] mRNA to obtain normalized amounts of transcripts), correlated significantly with proliferation (MIB-1), but with a significant negative correlation with apoptotic index (single-stranded DNA).

In hepatocellular carcinoma, a high survivin mRNA GAPDH ratio by RT-PCR is shown to correlate significantly with decreased disease-free 5 -year survival and increased recurrence rate. ${ }^{12,15}$ Also, the mean mRNA survivin:GAPDH ratio is significantly higher in patients with recurrence than in those without recurrence, ${ }^{12}$ a high ratio correlating with a high recurrence rate, ${ }^{16}$ and being a strong independent parameter of tumor recurrence after hepatectomy. ${ }^{12,16}$

We studied survivin expression by immunohistochemistry in a tissue microarray of hepatocellular carcinoma, correlating results with prognostic parameters, proliferation (MIB-1), recurrence rates, and outcome (overall and disease-free survival).

\section{Materials and methods}

\section{Tissue Specimens}

In total, 87 cases of hepatocellular carcinoma diagnosed between 1985 and 2002 were studied. These included 28 liver explants, 28 lobectomies, 14 segmentectomies, 12 wedge resections, and five autopsy specimens. All cases with pre- and postoperative treatment were excluded. The non-neoplastic liver studied, included six in cirrhotic liver adjacent to hepatocellular carcinoma, one in noncirrhotic liver with hepatocellular carcinoma, and one from a normal liver without cirrhosis or carcinoma. The hepatocellular carcinoma were histologically graded as I-III on the basis of cellular atypia and architectural complexity, according to Edmondson and Steiner. ${ }^{17}$ Clinical and macroscopic and/or microscopic features of adjacent non-neoplastic livers were used to diagnose cirrhosis. Nuclear grade was assessed as mild, moderate, or severe cytologic nuclear atypia, mitotic count was per 10 high-power fields $\times 400$ magnification (total area $1.5 \mathrm{~mm}^{2}$, with a single field of $0.152 \mathrm{~mm}^{2}(\times 40$ objective, $0.44 \mathrm{~mm}$ field diameter)), tumor necrosis, and microvascular invasion as present or absent. Staging (AJCC), ${ }^{18}$ tumor size, mode of therapy, and survival data were obtained by review of surgical pathology and/or autopsy reports, and from the
Winship Cancer Center database, with approval of the Investigational Review Board. The mean followup time was $23 \pm 20$ months.

Targeted tissue areas of 87 hepatocellular carcinoma and eight normal livers were marked on hematoxylin- and eosin-stained sections. Corresponding formalin-fixed paraffin-embedded tissue blocks were obtained from the archives of the Division of Anatomic Pathology, Department of Pathology and Laboratory Medicine, Atlanta, GA, with Institutional Review Board approval. Two tissue cores, $1.0 \mathrm{~mm}$ in diameter and $3-4 \mathrm{~mm}$ in thickness, were removed from each block, using a manual microarray device (Beecher Instruments, Silver Spring, MD, USA), with a total of 190 tissue cores inserted into the recipient paraffin block. The tissue microarray was sectioned at $4 \mu \mathrm{m}$ thickness and placed on charged slides.

Immunohistochemistry was performed after steam heat-induced epitope retrieval in a pressure cooker, using polyclonal survivin (1:80) (Santa Cruz Biotechnology, Santa Cruz, CA, USA) and monoclonal MIB-1 (clone MIB-1) (1/160) (DAKO Corporation, Carpinteria, CA, USA). An avidin-biotin complex kit (LSAB + System; DAKO) was used with the DAKO Autostainer. Hematoxylin was the counterstain. Sections of colonic adenocarcinoma were positive controls for survivin, and tonsil for MIB-1. Negative controls had primary antibody replaced by buffer. The survivin antibody used has been shown to react specifically with survivin of human origin by Western blotting and immunohistochemistry. Immunostain was recorded as $\mathbf{0}-3+$ according to stain intensity, distribution in cytoplasm and/or nucleus, and percentage of cancerous cells that stained positively. Tumors were scored on a fourtiered system, with $<10 \%$ of carcinoma cells staining called negative, $10-25 \%$ positively scored as $1,26 \%-50 \%$ scored as $2,51-75 \%$ scored as 3 , and 76-100 scored as 4 .

\section{Statistical Analysis}

The data were analyzed using a combination of Fisher's exact test, $t$-tests, analysis of variance (ANOVA), Pearson's correlation, and Kaplan-Meier survival curves followed by logrank tests and Cox proportional hazards modes.

When comparing two categorical factors such as nuclear grade vs cirrhosis, Fisher's exact tests were used. When comparing a dichotomous factor vs a continuous factor such as nuclear stain vs tumor size, $t$-tests were used. When comparing a categorical factor vs continuous factor such as nuclear stain at three levels vs tumor size, ANOVA followed by Tukey's pairwise comparisons were used. Pearson's correlation was used to test the relationship between two continuous factors such as MIB-1 and nuclear stain percent. 
Finally, overall and disease-free survival were compared across other factors using Kaplan-Meier curves and logrank tests. For those factors that were continuous, such as size, Cox proportional hazards model was used instead.

\section{Results}

In all, $72(83 \%)$ of the 87 cases of hepatocellular carcinoma were adequate for evaluation. The median age was 59 years (range 24-84). Totally, 77\% of patients were male and $23 \%$ female. Cirrhosis was present in $61 \%$. The median tumor size was $4.5 \mathrm{~cm}$ (range 0.4-19); the mean tumor size $5.5 \pm 4.4 \mathrm{~cm}$. In total, $72 \%$ of the hepatocellular carcinoma were solitary tumors, $28 \%$ multiple. Regional nodes were positive for metastatic hepatocellular carcinoma in three of the 72 cases. Metastases were identified in $15 \%$. Overall, $28 \%$ were Stage I, $43 \%$ Stage II, $12 \%$ Stage III, and $18 \%$ Stage IV. Local recurrences occurred in $15 \%$.

Histopathologic types were 69, not otherwise specified, two fibrolamellar and one mixed hepatocellular carcinoma/cholangiocarcinoma. Histologic grade was I in $12.5 \%$, II in $75 \%$, and III in $12.5 \%$. Nuclear grade was 1 in $18 \%, 2$ in $50 \%$, and 3 in $32 \%$. Tumor necrosis was present in $54 \%$, microvascular invasion in 54\%. Nuclear survivin expression was present in $43 \%$ of 87 hepatocellular carcinoma (Figure 1a), but in none of the eight normal livers (Figure 1b). The mean mitotic activity was 2.8 per 10 high-power fields. Mean MIB-1 labeling index was $6.1 \%$ in hepatocellular carcinoma (Figure 1c), and $<2 \%$ in normal livers (Figure 1d).

Table 1 shows the correlation of nuclear survivin immunopositivity in the hepatocellular carcinoma with clinicopathologic parameters. Survivin immunoreactivity in the hepatocellular carcinoma correlated significantly with nuclear grade $(P=0.0271)$, microvascular invasion $(P=0.0064)$, and local recurrence $(P=0.0487)$. There was a tendency for Stage with Stages III and IV grouped together $(P=0.0548)$, and histologic grade $(P=0.0544)$ to correlate with nuclear survivin expression. Other parameters including tumor necrosis, showed no correlation with survivin expression.

Comparing the mean number of mitoses per 10 high-power fields and the mean MIB-1 labeling index in hepatocellular carcinoma with and without nuclear survivin expression (Table 2), there was as significant correlation with the presence of survivin $(P=0.0017$ and 0.0001 , respectively). Figure 2 graphs nuclear survivin expression against MIB-1 labeling index $(P=0.0001$, correlation coefficient $R=0.526$ ).

Disease-free survival was significantly better in patients with hepatocellular carcinoma lacking survivin expression, as compared with those whose hepatocellular carcinoma expressed survivin
$(P=0.0098)$ (Figure 3a). This improvement in disease-free survival is also noted when comparing patients with no $(<10 \%$ positive) vs low vs high survivin immunoreactivity $(P=0.026)$ in their neoplasms (Figure $3 \mathrm{~b}$ ). The breakdown into survivin expression low (10-15\% positive) and high ( $>15 \%$ positive) was based on median split. The overall survival did not correlate with survivin expression $(P=0.4994)$.

By multivariate analysis, tumor size was the only parameter related to overall survival $(P=0.0033)$, while MIB-1 was the most important factor related to disease-free survival $(P=0.0006)$.

\section{Discussion}

Hepatocellular carcinoma is the fifth most common malignancy in men, and the eighth in women, accounting for 1 million deaths in the world annually. ${ }^{19,20}$ Risk factors include cirrhosis, hepatitis $\mathrm{B}$ and $\mathrm{C}$ infections, alcohol abuse, aflatoxin exposure, hemochromatosis, and alpha-1-antitrypsin deficiency. ${ }^{19,21}$ The only definitive treatment is surgical resection or transplantation, ${ }^{19,20}$ although over half the patients have disease recurrence after surgical resection. ${ }^{19}$ Attempts have been made to identify markers that can predict disease recurrence. Poor clinicopathologic prognostic factors include TNM staging (size, multiplicity, nodal status, metastasis, microvascular invasion $)^{18,20}$ and nuclear grade and microvascular invasion. ${ }^{22}$

Survivin expression, nuclear in distribution by immunohistochemistry, and/or by RT-PCR is reported to be associated with unfavorable histology and aggressive disseminated disease in endometrial and ovarian carcinoma. ${ }^{10,23}$ The overall survival in pulmonary non-small-cell carcinoma, ${ }^{24}$ esophageal carcinoma, ${ }^{25,26}$ gliomas, ${ }^{27}$ soft-tissue sarcoma, ${ }^{28}$ squamous laryngeal carcinoma, ${ }^{29}$ and endometrial carcinoma $^{10}$ is significantly decreased in patients with neoplasms expressing survivin as compared to those with no expression. Disease-free survival is significantly decreased in patients with malignant melanoma, ${ }^{30}$ and recurrence rate is increased in patients with neuroblastoma ${ }^{31}$ when survivin is present in the neoplasm.

In hepatocellular carcinoma patients, disease-free survival is decreased, ${ }^{12}$ and the recurrence rate increased, ${ }^{12}$ when survivin is demonstrated in the carcinoma by RT-PCR. We, using immunohistochemistry, show nuclear survivin expression in hepatocellular carcinoma to correlate with poor prognostic parameters (nuclear grade, microvascular invasion, and proliferation as mitotic rate and MIB-1 labeling index) and worse outcome (shorter diseasefree survival, increased recurrence rate).

Survivin expression is reported in $41 \%$ hepatocellular carcinoma by mRNA $^{12}$ concordant with immunohistochemistry. Our $43 \%$ frequency of nuclear survivin by immunohistochemistry in 

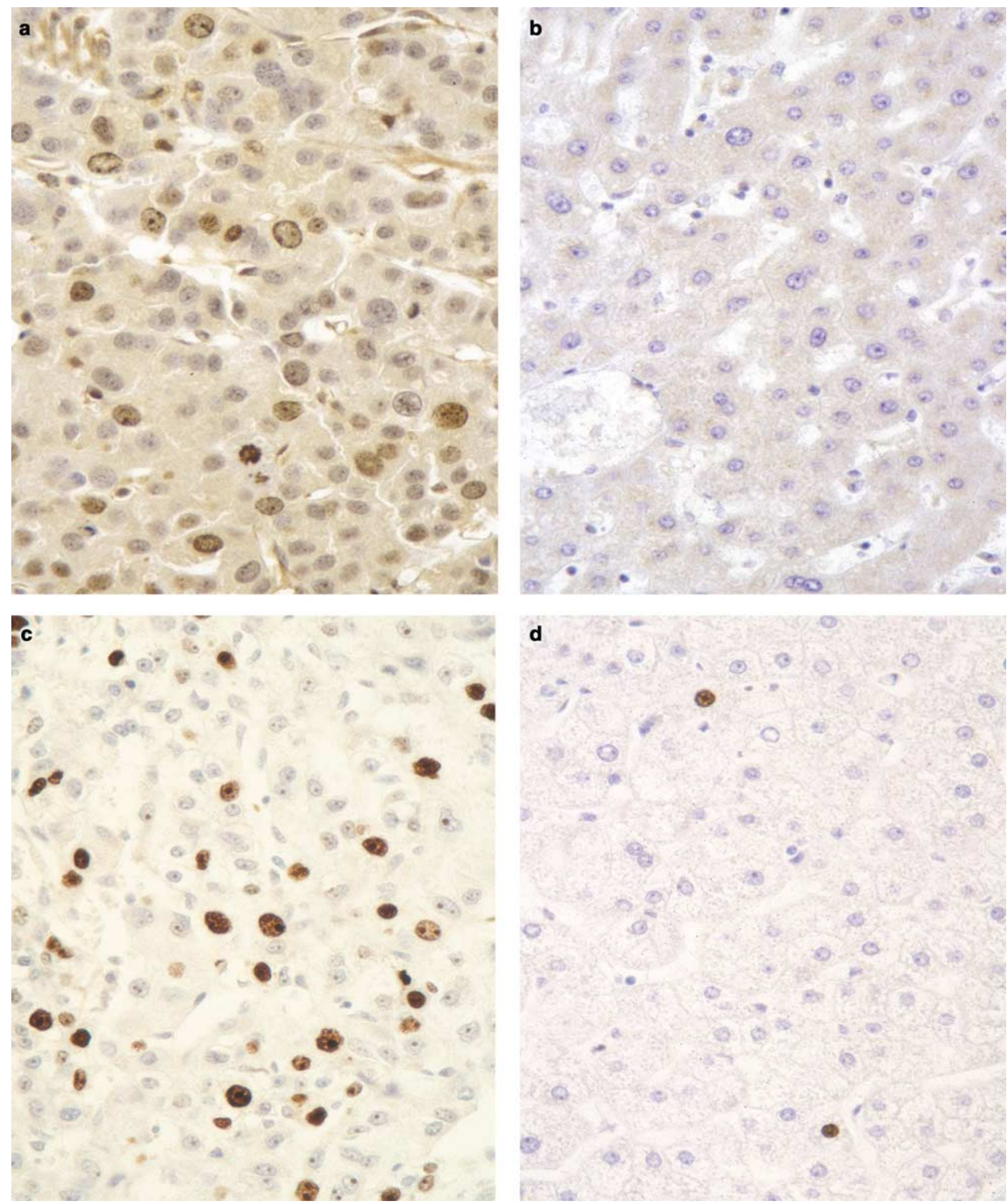

Figure 1 Nuclear survivin expression in hepatocellular carcinoma (a), but its absence in non-neoplastic liver (b), high-proliferation (nuclear MIB-1) in hepatocellular carcinoma (10\%) (c), and low-proliferation $(<2 \%)$ in non-neoplastic liver (d).

hepatocellular carcinoma is similar, although both are lower than the $63 \%$ expression by immunohistochemistry ${ }^{32}$ and the $70 \%$ expression by immunofluorescence previously reported. ${ }^{13}$ None of the non-neoplastic livers in our study showed survivin expression, similar to the $0 \%$ in six normal livers and $4 \%$ (two of 51) in noncancerous liver tissue adjacent to hepatocellular carcinoma reported by 
Table 1 Correlation of nuclear survivin positivity in 87 hepatocellular carcinoma according to clinicopathologic parameters

\begin{tabular}{|c|c|c|c|}
\hline Variable & Category & Survivin pos (\%) & $\mathrm{P}$-value \\
\hline \multirow[t]{2}{*}{ Gender } & Male & $21 / 55(38 \%)$ & \multirow[t]{2}{*}{0.4550} \\
\hline & Female & $10 / 17(59 \%)$ & \\
\hline \multirow[t]{2}{*}{ Cirrhosis } & Present & $17 / 44(39 \%)$ & \multirow[t]{2}{*}{0.4220} \\
\hline & Absent & $14 / 28(50 \%)$ & \\
\hline \multirow{2}{*}{ Tumor number } & Solitary & $22 / 54$ (41\%) & \multirow[t]{2}{*}{0.5794} \\
\hline & Multiple & $9 / 18(50 \%)$ & \\
\hline \multirow[t]{4}{*}{ TNM stage } & Stage I & $5 / 19(26 \%)$ & \multirow[t]{4}{*}{0.0695} \\
\hline & Stage II & $13 / 29(45 \%)$ & \\
\hline & Stage III & $4 / 8(50 \%)$ & \\
\hline & Stage IV & $9 / 12(75 \%)$ & \\
\hline \multirow[t]{3}{*}{ TNM stage } & Stage I & $5 / 19(26 \%)$ & \multirow[t]{3}{*}{0.0548} \\
\hline & Stage II & $13 / 29(45 \%)$ & \\
\hline & Stage III, IV & $13 / 20(65 \%)$ & \\
\hline \multirow[t]{3}{*}{ Histologic grade } & 1 & 1/9 (11\%) & \multirow[t]{3}{*}{0.0544} \\
\hline & 2 & $24 / 54(44 \%)$ & \\
\hline & 3 & $6 / 9(67 \%)$ & \\
\hline \multirow[t]{3}{*}{ Nuclear grade } & 1 & 2/13 (15\%) & \multirow{3}{*}{0.0271} \\
\hline & 2 & 15/36 (42\%) & \\
\hline & 3 & 14/23 (61\%) & \\
\hline \multirow{2}{*}{ Tumor necrosis } & Present & $11 / 22(50 \%)$ & \multirow[t]{2}{*}{0.4360} \\
\hline & Absent & $20 / 50(40 \%)$ & \\
\hline \multirow[t]{2}{*}{ MVI } & Present & $21 / 37$ (57\%) & \multirow[t]{2}{*}{0.0064} \\
\hline & Absent & $10 / 35(28 \%)$ & \\
\hline \multirow[t]{2}{*}{ Local recurrence } & Present & 9/11 (82\%) & \multirow[t]{2}{*}{0.0487} \\
\hline & Absent & 22/61 (36\%) & \\
\hline
\end{tabular}

$\mathrm{MVI}=$ microvascular invasion.

Ikeguchi et $a l^{12}$ with mRNA, and confirmed by immunohistochemistry, and the $0 \%$ in eight nontumorous livers with mRNA by Ito et al. ${ }^{13}$ Moon and Tarnawski $^{32}$ report only cytoplasmic stain in nonmalignant hepatocytes. Similar to the mean $6.1 \%$ MIB-1 labeling index in hepatocellular carcinoma and the $<2 \%$ mean in normal livers, we previously found by quantitative image cytometry, a mean MIB1 of $5.14 \%$ in low-grade and $8.06 \%$ in high-grade hepatocellular carcinoma. This is compared to $1.64 \%$ in normal liver without hepatocellular carcinoma and $2.17 \%$ in cirrhotic liver with hepatocellular carcinoma. ${ }^{33}$ Similarly, Pizem et $a l^{14}$ report significantly different mean Proliferation Nuclear Cell Antigen and Ki-67 proliferative indices of 7.41 and $9.67 \%$ in hepatocellular carcinoma, and 0.21 and $0.19 \%$ in non-neoplastic liver. They find significant differences in proliferation (Proliferation Nuclear Cell Antigen index) between different grades of hepatocellular carcinoma.

In the literature, cytoplasmic expression of survivin by immunohistochemistry is reported to be a poor prognostic parameter in neuroblastoma, ${ }^{34}$ laryngeal squamous cell, ${ }^{29}$ colorectal, ${ }^{2,5,35,36}$ and
Table 2 Correlation of mitotic count and proliferation (MIB-1) with nuclear survivin expression in hepatocellular carcinoma

Survivin (+) Survivin (-) P-value

\begin{tabular}{llll}
\hline Mitosis per $10 \mathrm{HPF}$, mean & $4.8(n=31)$ & $1.2(n=41)$ & 0.0017
\end{tabular} MIB-1 (\%) $\quad 9.6 \%(n=30) \quad 2.7 \%(n=41) \quad 0.0001$

$\mathrm{HPF}=$ high-power fields; MIB-1 (\%)= mean labeling index.

Survivin vs MIB-1 expression

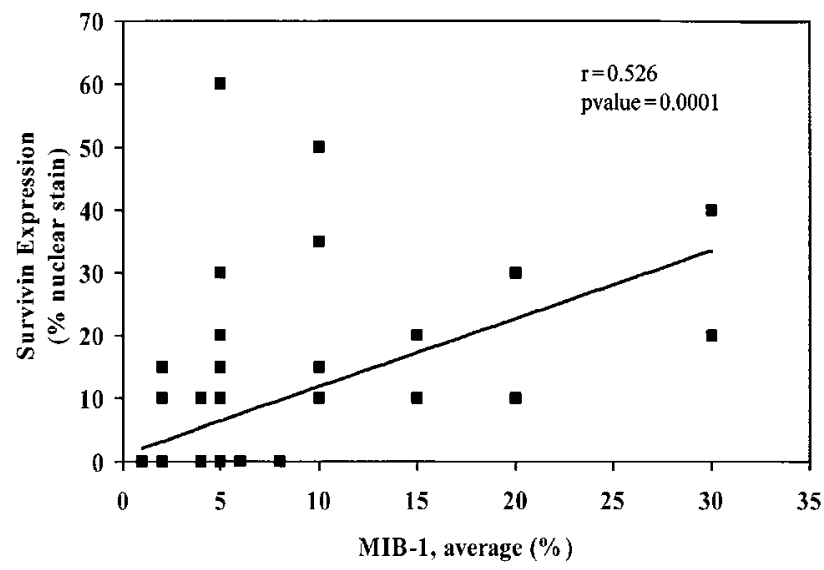

Figure 2 Nuclear survivin expression in hepatocellular carcinoma correlates significantly $(P=0.0001)$ with proliferation (MIB-1) with correlation coefficient $R=0.526$.

urothelial $^{37}$ carcinoma. For pancreatic, ${ }^{38}$ gastric, ${ }^{39,40}$ esophageal, ${ }^{26}$ and urothelial $^{41}$ carcinomas, on the other hand, no association was found between cytoplasmic survivin and patient survival. The translocation of survivin from cytoplasmic in the normal to cytoplasmic and nuclear in high-grade dysplasia and squamous cell carcinoma is noted in the esophagus. ${ }^{26}$ The differential nuclear and cytoplasmic localization of survivin is shown to be due to differences in the amino-acid sequence of its carboxy-terminal domain. ${ }^{26}$ In hepatocellular carcinoma, the predominant function of survivin is its cell cycle nuclear distribution, and not the cytoplasmic caspase-3-dependent antiapoptotic effect. ${ }^{13}$ Thus, mean proliferation labeling index (Ki-67, Proliferation Cell Nuclear Antigen) is shown to correlate significantly with survivin, whereas apoptotic index (single-stranded DNA immunohistochemistry, Tunel) does not. ${ }^{13-16}$ This suggests that, as in hepatocellular carcinoma, the prognostic significance in other cancers of survivin immunostain in nuclei relates to its cell cycle, and not to its cytoplasmic antiapoptotic, effect. Moon and Tarnawski $^{32}$ suggest that cytoplasmic localization of survivin in quiescent nonmalignant cells suppresses apoptosis, while its nuclear translocation in hepatocellular carcinoma may be important in regulation of proliferation and differentiation. 
Disease-Free Survival

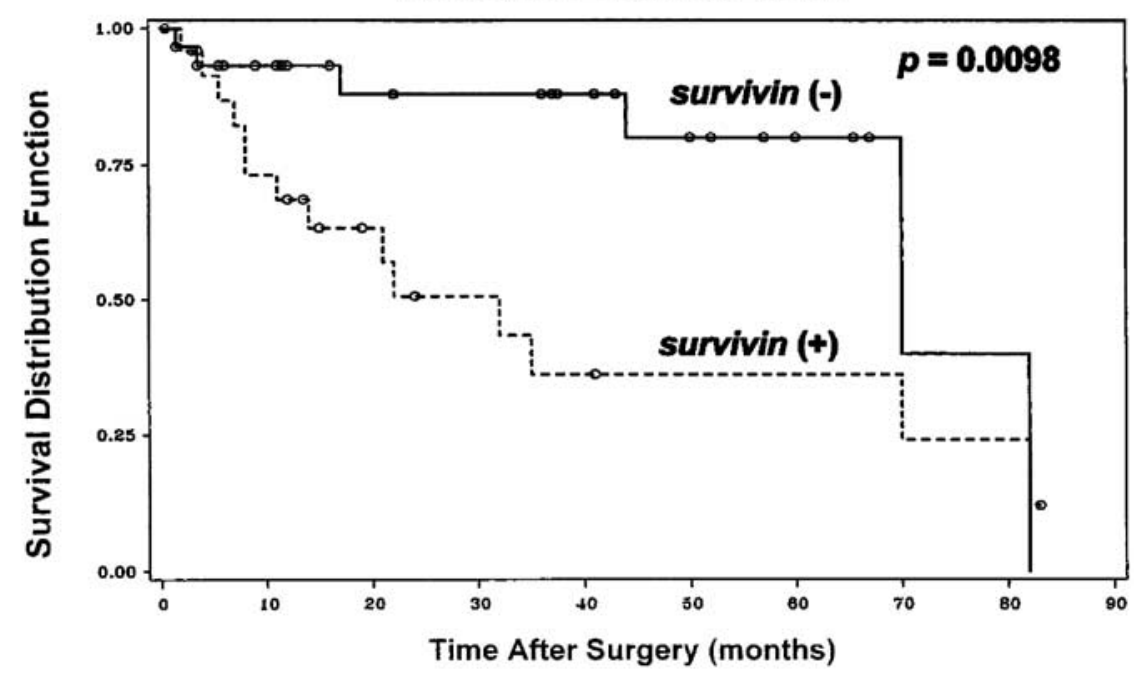

b

Disease-Free Survival

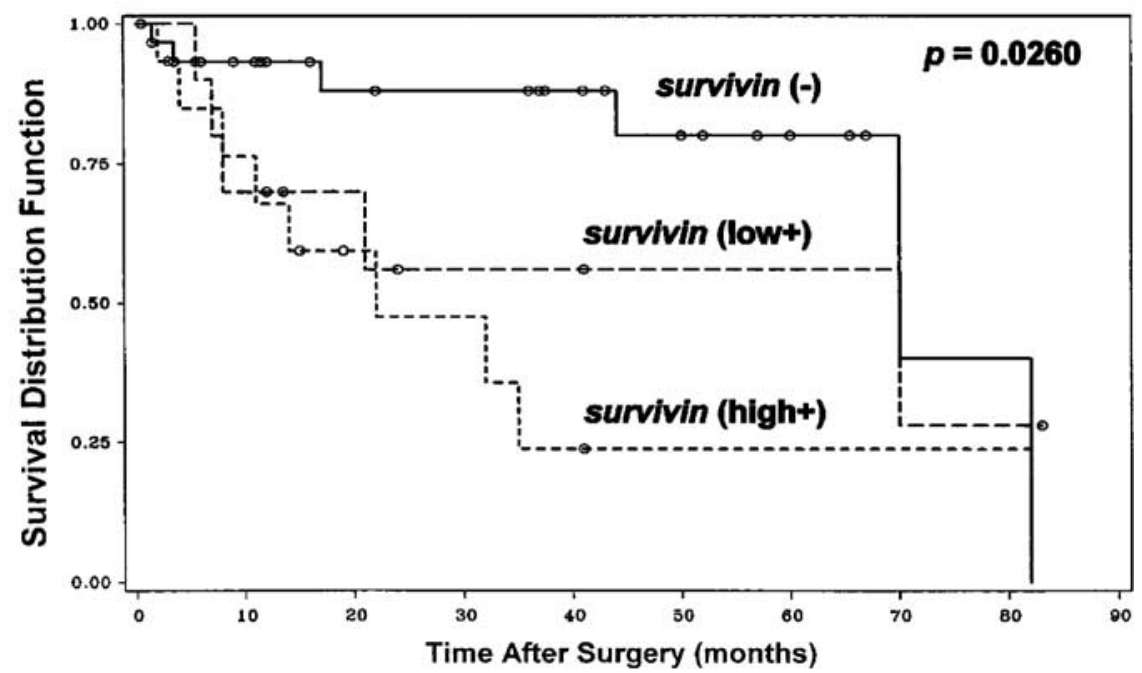

Figure 3 Disease-free survival is significantly less $(P=0.0098)$ in patients with hepatocellular carcinoma immunopositive ( $\geq 10 \%)$ for nuclear survivin $(a)$; this significance $(P=0.0260)$ remains when patients are subdivided according to high $(>15 \%)$ and low $(10-15 \%)$ survivin expression in their tumors, with disease-free survival worse when survivin expression is high (b).

Ikeguchi et $a l^{16}$ show a significant difference in mean Ki-67 labeling index according to histologic differentiation (grade), but not with size or stage of the hepatocellular carcinoma. They, Ito et $a 1^{13}$ and Pizem et $a l^{14}$ report that, in hepatocellular carcinoma, the mean proliferation index did correlate significantly with survivin, which did not correlate with apoptotic index. In several other cancers (ovarian, ${ }^{42}$ esophageal, ${ }^{26}$ pancreatic, ${ }^{38}$ colorectal $^{43}$ ), survivin overexpression correlates with increased proliferation, although the expression was cytoplasmic in the pancreatic and colorectal carcinomas. Survivin gene targeting inhibits cell proliferation, and induces apoptosis in in vitro cell lines. ${ }^{44}$

Our immunohistochemical study in hepatocellular carcinoma confirms the presence of nuclear survivin in $43 \%$ of the neoplasms, its expression correlating with poor clinicopathologic parameters (stage, histologic and nuclear grade, microvascular invasion, mitotic activity, MIB-1 proliferation index), local recurrence and disease-free survival. Anti-survivin therapies may not only potentiate chemotherapies that stimulate apoptosis, but, by removal of the antiapoptotic effect of survivin, may also exert an anti proliferative effect.

\section{Acknowledgement}

We thank Ms Judy Dunbar for secretarial support. The paper was presented at the United States and 
Canadian Academy of Pathology Annual Meeting, Washington, DC, March, 2003.

\section{References}

1 Deveraux QL, Reed JC. IAP family proteins-suppressors of apoptosis. Genes Dev 1999;13:239-252.

2 LaCasse EC, Baird S, Korneluk RG, et al. The inhibitors of apoptosis (IAPs) and their emerging role in cancer. Oncogene 1998;17:3247-3259.

3 Ambrosini G, Adida C, Altieri DC. A novel antiapoptosis gene, survivin, expressed in cancer and lymphoma. Nat Med 1997;3:917-921.

4 Altieri DC. The molecular basis and potential role of survivin in cancer diagnosis and therapy. Trends Mol Med 2001;7:542-547.

5 Altieri DC, Marchisio C. Survivin apoptosis; an interloper between cell death and cell proliferation in cancer. Lab Invest 1999;79:1327-1333.

6 Reed JC. The survivin saga goes in vivo. J Clin Inves 2001;108:965-969.

7 Geske FJ, Gerschenson LE. The biology of apoptosis. Hum Pathol 2001;32:1029-1038.

8 Saikumar P, Dong Z, Mikhailov W, et al. Apoptosis: definition, mechanisms, and relevance to disease. Am J Med 1999;107:489-506.

9 Tamm I, Wang Y, Sausville E, et al. IAP-family protein survivin inhibits caspase activity and apoptosis induced by Fas (CD95), bax, caspases, and anticancer drugs. Cancer Res 1998;58:5315-5320.

10 Takai N, Miyazaki T, Nishida M, et al. Survivin expression correlates with clinical stage, histological grade, invasive behavior, and survival rate in endometrial carcinoma. Cancer Lett 2002;184:105-116.

11 Li F, Ambrosini G, Chu EY. Control of apoptosis and mitotic spindle checkpoint by survivin. Nature 1998;396:580-584.

12 Ikeguchi M, Ueda T, Sakatani T, et al. Expression of survivin messenger RNA correlates with poor prognosis in patients with hepatocellular carcinoma. Diagn Mol Pathol 2002;11:33-40.

13 Ito T, Shiraki K, Sugimoto K, et al. Survivin promotes cell proliferation in human hepatocellular carcinoma. Hepatology 2000;31:1080-1085.

14 Pizem J, Marolt VF, Luzar B, et al. Proliferative and apoptotic activity in hepatocellular carcinoma and surrounding non-neoplastic liver tissue. Pflugers Arch 2001;442(6 Suppl): R174-R176.

15 Ikeguchi M, Ueta T, Yomane Y, et al. Inducible nitric oxide synthase and survivin messenger RNA expression in hepatocellular carcinoma. Clin Cancer Res 2002;8:3131-3136.

16 Ikeguchi M, Hirooka Y, Kaibara N. Quantitative analysis of apoptosis-related gene expression in hepatocellular carcinoma. Cancer 2002;95:1938-1945.

17 Edmondson HA, Steiner PE. Primary carcinoma of the liver: a study of 100 cases among 48,900 necropsies. Cancer 1954;7:462-503.

18 Liver (including intrahepatic bile ducts), American Joint Committee on Cancer, AJCC Cancer Staging Manual, 6th edn. Springer-Verlag: New York, NY, 2002, pp 131-138.

19 Hassoun Z, Gores GJ. Treatment of hepatocellular carcinoma. Clin Gastroenterol Hepatol 2003;1:10-18.
20 Befeler AS, Bisceglie AM. Hepatocellular carcinoma: diagnosis and treatment. Gastroenterology 2002; 122:1609-1619.

21 Tran TT, Poordad FF, Nissen N, et al. Hepatocellular carcinoma: an update. Clin Persp Gastroenterol 2002, Sept., Oct.: 302-306 (No Volume available).

22 Lauwers GY, Terris B, Balis UJ, et al. Prognostic histologic indicators of curatively resected hepatocellular carcinomas: a multi-institutional analysis of 425 patients with definition of a histologic prognostic index. Am J Surg Pathol 2002;26:25-34.

23 Cohen C, Lohmann CM, Cotsonis G, et al. Survivin expression in ovarian carcinoma: correlation with apoptotic markers and prognosis. Mod Pathol 2003;16:574-583.

24 Monzo M, Rosell R, Felip E, et al. A novel antiapoptosis gene: re-expression of survivin messenger RNA as a prognosis marker in non-small cell lung cancers. J Clin Oncol 1999;17:2100-2104.

25 Kato J, Kuwabara Y, Mitani M, et al. Expression of survivin in esophageal cancer: correlation with the prognosis response to chemotherapy. Int J Cancer 2001;95:92-95.

26 Grabowski P, Kuhnel T, Muhr-Wilkenshoff F, et al. Prognostic value of nuclear survivin expression in oesophageal squamous cell carcinoma. Br J Cancer 2003;88:115-119.

27 Chakravarti A, Noll E, Black PM, et al. Quantitatively determined survivin expression levels are of prognostic value in human gliomas. J Clin Oncol 2002; 20:1063-1068.

28 Kappler M, Kohler T, Kampf C, et al. Increased survivin transcript levels: an independent negative predictor of survival in soft tissue sarcoma patients. Int J Cancer 2001;95:360-363.

29 Dong Y, Sui L, Watanabe Y, et al. Survivin expression in laryngeal squamous cell carcinomas and prognostic implications. Anticancer Res 2002;22: 2377-2384.

30 Gradilone A, Gazzaniga P, Ribuffo D, et al. Survivin, $b c l-2, b a x$, and $b c l-x$ gene expression in sentinel lymph nodes from melanoma patients. J Clin Oncol 2003;21:306-312.

31 Azuhata T, Scott D, Takamizawa S, et al. The inhibitor of apoptosis protein survivin is associated with highrisk behavior of neuroblastoma. J Pediatr Surg 2001;36:1785-1791.

32 Moon WS, Tarnawski AS. Nuclear translocation of survivin in hepatocellular carcinoma: a key to cancer cell growth? Hum Pathol 2003;34:1119-1126.

33 Wells SJ, DeRose PB, Cohen C. Image cytometric comparison of proliferating cell nuclear antigen and MIB-1 staining in hepatocellular carcinoma and adjacent liver tissue. Cytometry 1996;26:198-203.

34 Adida C, Berrebi D, Peuchmaur M, et al. Antiapoptosis gene, survivin, and prognosis of neuroblastoma. Lancet 1998;351:882-883 (letter).

35 Kawasaki H, Altieri DC, Lu CD, et al. Inhibition of apoptosis by survivin predicts shorter survival rates in colorectal cancer. Cancer Res 1998;58:5071-5074.

36 Sarela AI, Scott N, Ramsdale J, et al. Immunohistochemical detection of the anti-apoptosis protein, survivin, predicts survival after curative resection of stage II colorectal carcinomas. Ann Surg Oncol 2001;8:305-310.

37 Swana HS, Grossman D, Anthony JN, et al. Tumor content of the anti-apoptosis molecule survivin and 
recurrence of bladder cancer. N Engl J Med 1999; $341: 452-453$.

38 Sarela AI, Verbeke CS, Ramsdale J, et al. Expression of survivin, a novel inhibitor of apoptosis and cell cycle regulatory protein, in pancreatic adenocarcinoma. Br J Cancer 2002;86:886-892.

39 Okada E, Murai Y, Matsui K, et al. Survivin expression in tumor cell nuclei is predictive of a favorable prognosis in gastric cancer patients. Cancer Lett 2001; 163:109-116.

40 Ikeguchi M, Kaibara N. Changes in survivin messenger RNA level during cisplatin treatment in gastric cancer. Int J Mol Med 2001;8:661-666.

41 Nakanishi K, Tominaga S, Hiroi S, et al. Expression of survivin does not predict survival in patients with transitional cell carcinoma of the upper urinary tract. Virchows Arch 2002;441:559-563.

42 Sui L, Dong Y, Ohno M, et al. Survivin expression and its correlation with cell proliferation and prognosis in epithelial ovarian tumors. Int J Oncol 2002;21: $315-320$

43 Kawasaki H, Toyoda M, Shinohara H, et al. Expression of survivin correlates with apoptosis, proliferation, and angiogenesis during human colorectal tumorigenesis. Cancer 2001;91: 2026-2032.

44 Ambrosini G, Adida C, Sirugo G, et al. Introduction of apoptosis and inhibition of cell proliferation by survivin gene targeting. J Biol Chem 1998;273: 11177-11182. 